\title{
Transient heat flux measurement using a surface junction thermocouple
}

\author{
S. R. Sanderson ${ }^{\text {a) }}$ and B. Sturtevant ${ }^{\text {b) }}$ \\ Graduate Aeronautical Laboratories, California Institute of Technology, Pasadena, California 91125
}

(Received 29 January 2002; accepted for publication 12 April 2002)

\begin{abstract}
A new form of surface junction thermocouple sensor has been developed and tested. The novel feature of the design is the use of a tapered fit between two coaxial thermocouple elements to form a thin, robust junction. The gauge has a response time on the order of $1 \mu$ s and is suitable for measuring large transient heat fluxes in hypervelocity wind tunnels. Asymptotic analysis is used to demonstrate the operating principles and to assess the errors associated with the finite thickness of the surface junction. Spectral deconvolution methods are used to infer a mean square optimal estimate of the surface heat flux from time resolved surface temperature measurements. This improved signal processing method is applicable to transient heat flux gauges of all types. Potential reducible error sources and other systematic errors are described. Measurements of the heat flux about the forebody of a cylindrical body in a hypervelocity flow demonstrate the functioning of the gauge and are used to obtain statistical estimates of the repeatability of the technique. The measured heat fluxes are compared with established theoretical predictions. (C) 2002 American Institute of Physics. [DOI: 10.1063/1.1484255]
\end{abstract}

\section{INTRODUCTION}

The measurement of heat transfer rates to aerodynamic surfaces is important in the design of hypervelocity aerospace vehicles. Hypervelocity flows may be simulated in laboratory wind tunnels for only a fraction of a second. High heat transfer rates result from the specific enthalpy of the flow and the elevated gas density required to achieve scaling of the relevant dimensionless parameters. Under these extreme conditions, the flow becomes contaminated with the products of mechanical, physical, and chemical breakdown of a variety of metallic and nonmetallic materials. Accurate heat transfer data are obtained only with difficulty in such an environment. The contribution of the current article is to describe new techniques that have proven to provide advantages in terms of accuracy, speed of data reduction and sensor reliability.

\section{BACKGROUND}

The operating principles, and typical sensor designs that are employed for transient heat transfer measurements are reviewed by Schultz and Jones. ${ }^{1}$ To obtain the fastest possible response from the sensor it is advantageous to base the heat transfer measurements on temperature data obtained directly at the surface of the body under study. The unsteady conduction of heat within a solid body is described by linear partial differential equations when the temperature gradients remain small. Thus the heat transfer rates to the surface of a body may be inferred from the time history of temperature measurements made close to the surface by solving the resulting inverse diffusion problem. Common sensing mecha-

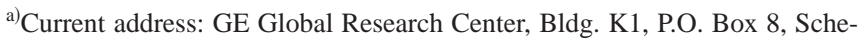
nectady, NY 12301; electronic mail: sanderson@crd.ge.com

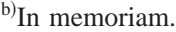

nisms for obtaining the required temperature data are the variation of resistivity of a thin metallic film with temperature or the generation of a thermoelectric electromotive force (emf) at a junction of dissimilar metals (see Schultz and Jones ${ }^{1}$ ). It is important to recognize that the choice of basic configuration has a significant impact on sensor degradation mechanisms, and thereby the accuracy and robustness of a sensor in hostile flow environments.

In order to elucidate the important operating principles and design criteria we briefly review the unsteady, linear conduction of heat in a one-dimensional semi-infinite solid that is described by

$$
\frac{\partial^{2} T(x, t)}{\partial x^{2}}=\frac{1}{\alpha} \frac{\partial T(x, t)}{\partial t},
$$

where $T$ is the temperature, $x$ is the spatial coordinate normal to the surface, $t$ is time, and $\alpha$ is the thermal diffusivity, $k$ is the thermal conductivity, $\rho$ is the density, $c$ is the specific heat of the material and the thermal diffusivity is $\alpha=k / \rho c$. For uniform initial condition $T_{\infty}$ and instantaneously applied, constant surface heat flux $\dot{q}_{0}$

$$
\begin{aligned}
& T(x, 0)=T_{\infty}, \\
& \left(\frac{\partial T}{\partial x}\right)_{x=0}=\frac{-\dot{q}_{0}}{k} ; \quad t>0,
\end{aligned}
$$

the solution is known to be

$$
\begin{aligned}
\Delta T=T-T_{\infty}= & \frac{2 \dot{q}_{0}}{k} \sqrt{\frac{\alpha t}{\pi}} \exp \frac{-x^{2}}{4 \alpha t} \\
& -\frac{\dot{q}_{0} x}{k}\left(1-\operatorname{erf} \frac{x}{2 \sqrt{\alpha t}}\right) .
\end{aligned}
$$




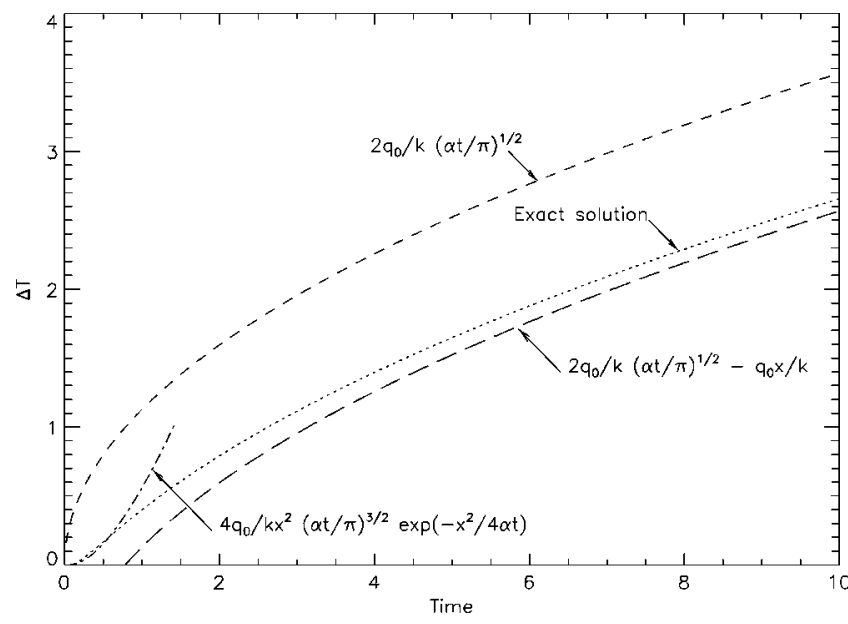

FIG. 1. Asymptotic behavior of a surface temperature sensor. For illustrative purposes $\alpha=1, k=1, \dot{q}_{0}=1$, and $x=1$.

Approximations to this exact solution are useful because they highlight the key design parameters for practical heat transfer gauges. Expanding this solution asymptotically in powers of $t$ as $t \rightarrow \infty$ at constant $x$ we obtain,

$$
\begin{aligned}
\Delta T= & 2 \frac{\dot{q}_{0}}{k} \sqrt{\frac{\alpha}{\pi}} t^{1 / 2}-\frac{\dot{q}_{0}}{k} x+\frac{1}{2} \frac{\dot{q}_{0} 1}{k \sqrt{\alpha \pi}} x^{2} t^{-(1 / 2)} \\
& +O\left(t^{-(3 / 2)}\right) .
\end{aligned}
$$

The first term is asymptotically valid for $\left[x /(\alpha t)^{1 / 2}\right]<1$ and expresses the usual result that the surface temperature rises parabolically with time for a constant heat flux. The criterion $\left[x /(\alpha t)^{1 / 2}\right]<1$ provides a limit on the required thinness of the surface sensing element for practical designs. The second order term $\left(\dot{q}_{0} / k\right) x$ gives the error in the surface temperature measurement associated with this finite thickness and this estimate is valid provided $\left[x /(\alpha t)^{1 / 4}\right]<1$.

Consider next the behavior at small times. Extracting an exponential prefactor and expanding asymptotically as $t$ $\rightarrow 0$ at constant $x$, the leading order behavior is

$$
\Delta T \asymp \frac{4 \dot{q}_{0}}{k x^{2}}\left(\frac{\alpha t}{\pi}\right)^{3 / 2} \exp \frac{-x^{2}}{4 \alpha t} ; \quad \frac{x}{(\alpha t)^{1 / 2}}>1 .
$$

Hence at small times the temperature rises exponentially. Observation of a satisfactorily rapid exponential rise in the surface temperature is indicative of the attainment of a sufficiently thin surface junction in practical gauges. The significance of the various terms of the expansion is illustrated in Fig. 1.

\section{PRACTICAL GAUGE DESIGNS}

A commonly used technique for gauge fabrication is to deposit a thin metal film (typically platinum) on a glass or ceramic substrate. The surface temperature is then sensed through the temperature dependence of the resistivity of the metal film. This technique yields a relatively sensitive device-however, the thin film is prone to damage in hostile environments. Any erosion of the metal film alters the sensitivity of the gauge. Calibration of each individual gauge is required except in the case where the resistance of the sen- sitive area of the film is much larger than the other elements of the gauge. If the metal is of sufficient purity, and the above condition is met, standard values of the resistivity as a function of temperature and the quiescent resistance of the gauge are sufficient to make a measurement of surface temperature.

An alternative physical mechanism for temperature measurement is the thermoelectric emf produced at a junction of dissimilar metals. If these materials are deposited on an insulating substrate, as described above, then the sensitivity of the gauge is a function of the substrate and thermocouple material properties only, and is unaffected by erosion of the sensor surface or the mechanical configuration of the gauge.

If a metallic substrate is used, the gauge is capable of tolerating a larger transient heat flux. The figure of merit at constant exposure duration and heat flux, as indicated by leading order of Eq. (5), is $T_{\max } \sqrt{\rho c k}$. Here $T_{\max }$ is the maximum working temperature for the material. A particularly robust design results when the thermocouple material itself is used as the substrate. The gauge is again unaffected by surface erosion and the sensitivity depends only on physical properties of the materials. Thus calibration of individual gauges is not required.

The design problem in producing such a metallic substrate gauge is to insulate the two conductors that form the thermocouple so that only a thin surface junction is formed. Common practice is to construct the gauge from coaxial cylindrical thermocouple elements that are insulated by a layer of epoxy or ceramic. A surface junction may be formed by electrolytic or vacuum deposition of a metal film, or by abrasion of the exposed surface to burr across the small gap. In either case very fine tolerances are required to produce a small gap between the thermocouple elements and the effective thickness of the junction scales with the width of the gap between the conductors. The allowable thickness is given by the condition $\left[x /(\alpha t)^{1 / 2}\right]<1$, and the associated error $\left(\dot{q}_{0} / k\right) x$ in the surface temperature measurement. Clearly as the test time is reduced, a smaller coaxial gap is required to maintain gauge performance.

\section{DESCRIPTION OF NEW GAUGE DESIGN}

A new gauge design has been developed and tested with the aim of simplifying the construction and improving the robustness of surface junction thermocouple sensors. The essential feature of this design is the use of a slightly tapered center conductor. The resulting interference between the tapered center pin and the sharp edged outer conductor forms a thin surface junction and provides reliable insulation (refer to Fig. 2). This design results in a robust transducer whose sensitivity is determined solely by the physical properties of the thermocouple materials. There is some weak dependence of sensitivity on the taper angle, however this is constant for all gauges and is estimable by calibration or analysis to any required degree of accuracy (e.g., Lyons and $\mathrm{Gai}^{2}$ ).

The effective thickness of the junction may be estimated from the surface roughness of the thermocouple components and the taper angle of the center conductor (refer to Fig. 3). The thickness of the junction, averaged over the two oppos- 


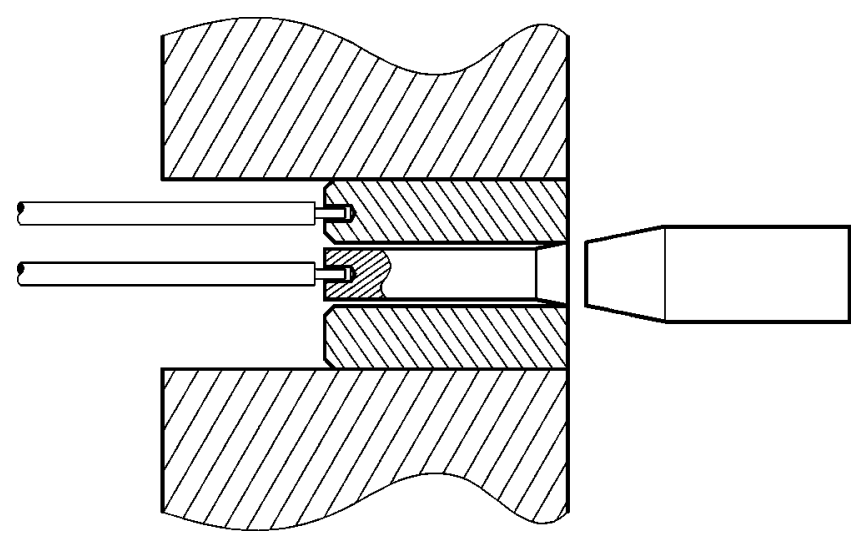

FIG. 2. Layout of new surface junction thermocouple sensor.

ing surfaces, may be estimated to be thickness=taper $\times$ roughness. It is possible to achieve a $0.3 \mu \mathrm{m}$ finish with conventional machining operations and with a 5:1 taper the average junction depth is $1.5 \mu \mathrm{m}$. For the gauges described here type $\mathrm{E}$ (Chromel-Constantan) thermocouples were used and the average thermal diffusivity for these materials is 5 $\times 10^{-6} \mathrm{~m}^{2} / \mathrm{s}$. A response time of $0.5 \mu$ s then follows from the condition $\left[x /(\alpha t)^{1 / 2}\right]<1$. The test time for typical hypervelocity wind tunnels lies in the range $1-100 \mathrm{~ms}$ and so this is acceptable. The length of the gauge must be sufficient such that the temperature rise at the rear surface is negligible. This is determined from the condition $\left[x /(\alpha t)^{1 / 2}\right] \gg 1$; here the relevant scales are understood to be the test time and the length of the gauge.

A simple differential amplifier eliminates common mode thermoelectric emf and reduces signal noise levels (see Fig. 4). The cold junction is formed at ambient temperature at the circuit board. This arrangement is adequate given the constancy of the cold junction temperature during the short run time and the large hot junction temperature rise experienced in hypervelocity wind tunnels (typically in the range 10-100 $\mathrm{K}$ for the experiments described here).

\section{DEDUCING THE SURFACE HEAT TRANSFER RATE}

If the unsteady conduction of heat in the thermocouple sensor is modeled by the analysis presented in Sec. II then, given the time history of the surface temperature and the physical properties of the gauge materials, it is possible to solve an inverse problem for the time history of the surface heat flux. Schultz and Jones ${ }^{1}$ describe procedures whereby the calculation is performed using a problem specific quadrature scheme or by an electronic analogue.

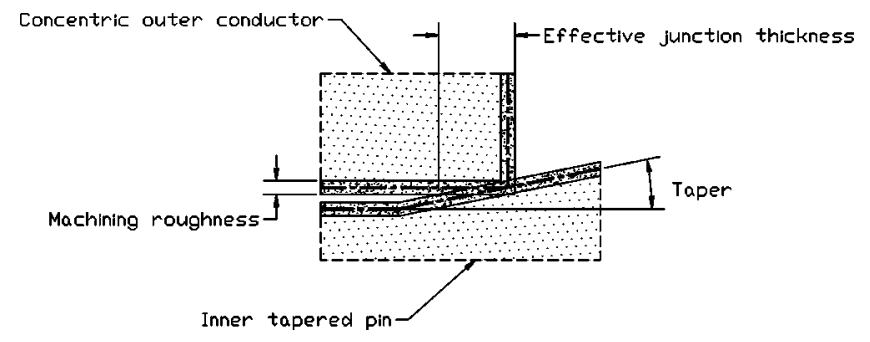

FIG. 3. Effective thickness of thermocouple junction.

Observe however that the governing equations are linear and hence the dynamic behavior of such a system may generally be described by the convolution integral

$$
\Delta T(x, t)=\int_{0}^{t} g(x, t-\tau) \dot{q}(\tau) d \tau .
$$

The unit impulse response function $g(x, t)$ is given by the derivative of Eq. (4)

$$
g(x, t)=\frac{\partial \Delta T(x, t)}{\partial t}=\sqrt{\frac{\alpha}{\pi k^{2} t}} \exp \frac{-x^{2}}{4 \alpha t} ; \quad t>0,
$$

and $x$ is the depth into the substrate at which the temperature is measured. Note that the integral is improper in the limit $x \rightarrow 0$ and a finite value of $x$ is taken to regularize the problem (characterized by the junction thickness as estimated in Sec. IV). Recasting the problem in this form allows the use of spectral deconvolution methods that provide a mean square optimal estimate of the time history of the surface heat flux. In comparison with the widely used techniques described by Schultz and Jones, ${ }^{1}$ the current approach provides advantages in terms of optimal rejection of noise and vastly increased computational speed. Theoretical background is given in Papoulis ${ }^{3}$ and the numerical algorithms required for efficient implementation using fast Fourier transform algorithms are described by Press et al. ${ }^{4} \mathrm{~A}$ concise description of these methods applied to an analogous problem is given by Sanderson and Simmons. ${ }^{5}$

Consider the time discretized form of the convolution integral Eq. (7)

$$
\Delta T_{i}=\sum_{k=0}^{i} g_{i-k} \dot{q}_{k} ; \quad i=0,1 \ldots N-1 .
$$

Here $g_{i}$ is the discretized unit impulse response, evaluated at the effective junction depth to regularize the problem. If the presence of noise is considered then

$$
z_{i}=\Delta T_{i}+n_{i},
$$

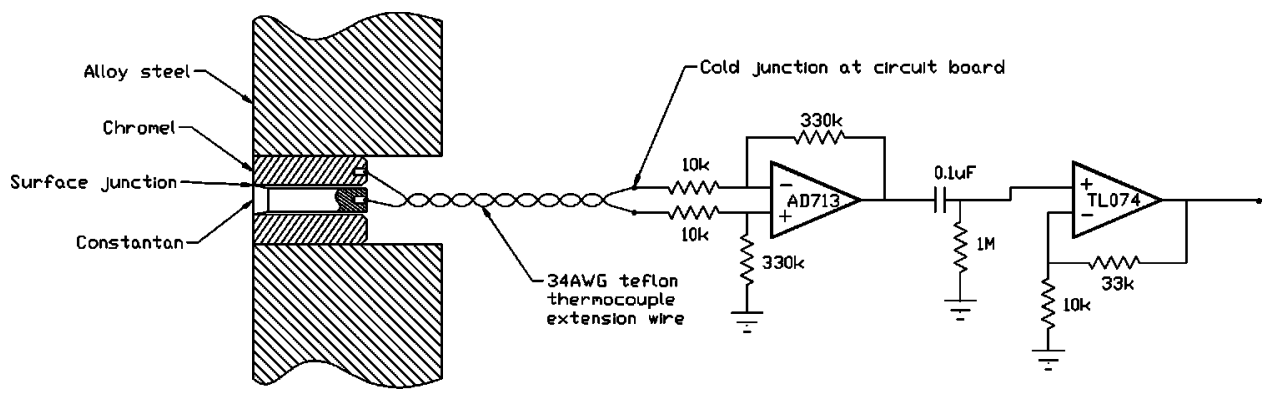

FIG. 4. Schematic of amplifier circuit. 
where $n_{i}$ is the signal noise and $z_{i}$ is the noisy data. The deconvolution problem comprises determining the filter $\phi_{i}$, which gives a least square optimal estimate $\dot{q}_{i}^{*}$ of the time history of the surface heat flux. Thus,

$$
\sum_{k=0}^{i} \phi_{i-k} z_{k}=\sum_{k=0}^{i} g_{i-k} \dot{q}_{k}^{*} ; \quad i=0,1 \ldots N-1 .
$$

By convolution property of the discrete Fourier transform, the frequency domain equivalent of Eq. (11) is

$$
\Phi_{j} Z_{j}=G_{j} \dot{Q}_{j}^{*} ; \quad j=0,1 \ldots N-1 .
$$

Upper case symbols denote the discrete Fourier transform of the corresponding lower case variable. By minimizing the variance of the estimation error, $\Sigma_{i=0}^{N-1}\left(\dot{q}_{i}-\dot{q}_{i}^{*}\right)^{2}$, with respect to the unknowns $\Phi_{j}$, it may be shown (e.g., Sanderson and Simmons, ${ }^{5}$ Press et al. ${ }^{4}$ ) that the optimum filter is approximated by

$$
\Phi_{j}=\frac{Z_{j} \bar{Z}_{j}-\nu_{j}}{Z_{j} \bar{Z}_{j}} .
$$

Here the over bar denotes the complex conjugate. Note that $Z \bar{Z}_{j}$ represents the power spectral density of the surface temperature measurement and the optimum filter is determined by its relationship to the noise power spectral density $\nu_{j}$. Finally, by inverse discrete Fourier transform, the optimal estimate of the surface heat flux is obtained

$$
\dot{q}_{i}^{*}=F^{-1}\left[\frac{\Phi_{j} Z_{j}}{G_{j}}\right] ; \quad i, j=0,1 \ldots N-1 .
$$

Note that all time series must be padded to twice their length with trailing zeroes before commencing any of the above operations. This negates the periodicity of the discrete Fourier transform that would otherwise corrupt the inherent causality of the convolution integral Eq. (7) (see e.g., Papoulis ${ }^{3}$ ).

\section{EXPERIMENTAL RESULTS}

We have conducted experiments using the new thermocouple sensors in the T5 free piston driver shock tunnel at GALCIT. This large hypervelocity test facility is capable of simulating the high density flows about space vehicles at speeds on the order of $5000 \mathrm{~m} / \mathrm{s}$. Flows at this high specific kinetic energy can only be produced in the laboratory for durations on the order of $2 \mathrm{~ms}$ before the surface temperature of the wind tunnel nozzle throat exceeds working limits. The rationale and operation of free piston shock tunnels are described by Stalker ${ }^{6}$ and Hornung et al. ${ }^{7}$ for the interested reader.

To demonstrate the performance of the new heat transfer gauge we have measured the stagnation point heat transfer rate experienced by a circular cylinder in hypervelocity flow. The cylinder had a diameter of $\phi 40 \mathrm{~mm}$ with aspect ratio 4.5. The model was equipped with a dense array of 24 thermocouples about the forebody. The sensitive area of the gauges subtended an angle of $2^{\circ}$ at the center of the cylinder and were spaced at $5^{\circ}$ intervals in the most densely instrumented area. The gauges were staggered in three planes to achieve maximum spatial resolution with the outer two planes offset $4.45 \mathrm{~mm}$ either side of the center. A cavity, internal to the model support, contained the thermocouple preamplifiers. This cavity and all components of the model were sealed from the external flow by O-rings and vented to atmosphere through pressure tubing attached to the rear of the model support. This ensured that the weakly ionized test gases did not interfere with gauge operation. Outputs from the preamplifiers were digitized and converted from thermoelectric potential to temperature using NIST standard correlations for type- $\mathrm{E}$ thermocouples with $1.7^{\circ} \mathrm{C}$ prescribed limits of error. Cold junction temperature was assumed to remain constant at ambient for the duration of testing.

Sensors were available to monitor all of the operating parameters of the T5 facility and determine the free stream conditions in the test section. The free stream conditions are listed in Table I. Test conditions were based on measurements of the initial shock tube fill pressure and temperature, incident shock speed prior to reflection, and supersonic nozzle reservoir pressure after shock reflection. The remaining test section parameters are determined from the directly measured quantities via one-dimensional (1D) inviscid gas dynamics including consideration of chemical nonequilibrium thermodynamics (refer to Refs. 8 and 9).

Figures 5 and 6 demonstrate the performance of the gauge when operated at the conditions listed in Table I. Figure 5 shows the time history of the supersonic nozzle reservoir pressure, the model stagnation point surface temperature, and the heat transfer rate deduced from it using the spectral deconvolution method described in Sec. V. The surface temperature is observed to rise according to a fractional power law with time and the deconvolved heat transfer rate remains approximately constant as expected. The surface temperature is observed to rise sharply at the point that it initially departs from the zero level. This indicates that a thin surface junction has been achieved [see Fig. 1 and Eq. (6) where the behavior on the shortest time scales is discussed]. A large spike appears in the deduced heat flux for times in the range $0.3-0.6 \mathrm{~ms}$. The spike is attributed to shock wave transients that are known to occur during the starting process of a shock tunnel nozzle. A characteristic sawtooth shape appears in the surface temperature record, coincident with the heat flux spike. The shape reflects the form of the surface temperature impulse response function [see Eq. (8)].

Figure 6 indicates the distribution of heat flux about the forebody of the cylinder for three different test conditions. The three test conditions span a range of stagnation enthalpies relative to the characteristic dissociation energy of the nitrogen test gas (Table I). The instantaneous heat transfer distribution is plotted for each of the test conditions along with average values taken over an interval of width $100 \mu$ s. Superimposed on the plots are bars representing the standard deviations of the time histories over the averaging interval. This gives an indication of the temporal fluctuations of the heat transfer rates at various locations on the model surface. Temporal variations in the tunnel free stream conditions cause variance in the individual gauge readings to be highly correlated. Indeed, at the instant shown for test condition A, all the instantaneous heat transfer data points lie below the corresponding average data. The final set of points on each 
TABLE I. Summary of test conditions. Test conditions were computed on the basis of measurements of the initial shock tube fill pressure and temperature, incident shock speed prior to reflection, and nozzle reservoir pressure after shock reflection. The dimensionless parameters for the ideal dissociating gas (IDG) model and stagnation point heat transfer model are defined in Sec. VII. Error estimates given for the fill conditions represent the accuracy of the pressure gauge and the variation of the ambient temperature. Error estimates for the measured shock speed and reservoir pressure are the standard deviation of the quantities sampled over the entire sequence of shots. The error in the computed quantities may be inferred from these estimates. Parameters for the IDG model are taken to be $\theta_{d}=113200 \mathrm{~K}, \rho_{d}=130000 \mathrm{~kg} \mathrm{~m}^{-3}$, and $m=14.0 \times 10^{-3} / 6.023$ $\times 10^{23} \mathrm{~kg}$.

\begin{tabular}{|c|c|c|c|c|}
\hline Test condition & & A & $\mathrm{B}$ & $\mathrm{C}$ \\
\hline \multirow[t]{4}{*}{$\begin{array}{l}\text { Shock tube } \\
\text { conditions }\end{array}$} & $\begin{array}{l}\text { Fill pressure } \\
(\mathrm{kPa} \pm 0.5 \mathrm{kPa})\end{array}$ & 75 & 32.5 & 20 \\
\hline & $\begin{array}{l}\text { Fill temperature } \\
(K \pm 4 \mathrm{~K})\end{array}$ & 297 & 297 & 297 \\
\hline & Shock speed $(\mathrm{m} / \mathrm{s})$ & $1890 \pm 100$ & $3500 \pm 245$ & $4360 \pm 255$ \\
\hline & Test gas & $\mathrm{N}_{2}$ & $\mathrm{~N}_{2}$ & $\mathrm{~N}_{2}$ \\
\hline \multirow{3}{*}{$\begin{array}{l}\text { Nozzle } \\
\text { reservoir } \\
\text { conditions }\end{array}$} & Pressure (MPa) & $14.6 \pm 0.7$ & $22.3 \pm 1.3$ & $28.3 \pm 1.9$ \\
\hline & Temperature (K) & 3210 & 7550 & 8960 \\
\hline & Enthalpy (MJ/kg) & 3.88 & 12.0 & 19.1 \\
\hline $\begin{array}{l}\text { Test section } \\
\text { conditions }\end{array}$ & $\begin{array}{l}\text { Velocity }(\mathrm{m} / \mathrm{s}) \\
\text { Density }\left(\mathrm{kg} / \mathrm{m}^{3}\right) \\
\text { Pressure }(\mathrm{kPa}) \\
\mathrm{N} \text { conc }(\mathrm{mole} / \mathrm{kg})\end{array}$ & $\begin{array}{l}2540 \\
0.0218 \\
1.03 \\
9.9 \times 10^{-5}\end{array}$ & $\begin{array}{l}4450 \\
0.0155 \\
5.48 \\
6.9 \times 10^{-1}\end{array}$ & $\begin{array}{l}5350 \\
0.0157 \\
11.4 \\
3.65 \times 10^{0}\end{array}$ \\
\hline $\begin{array}{l}\text { Test section } \\
\text { dimensionless } \\
\text { parameters }\end{array}$ & $\begin{array}{l}P_{\infty} \\
H_{0_{\infty}} \\
\alpha_{\infty} \\
\hat{\rho}_{d} \\
\operatorname{Re}_{D} \\
\hat{\rho}_{e} \\
\alpha_{e} \\
\mathrm{St}\end{array}$ & $\begin{array}{l}7.32 \times 10^{-3} \\
0.102 \\
1.4 \times 10^{-6} \\
6.0 \times 10^{6} \\
222000 \\
6.61 \\
2.0 \times 10^{-6} \\
0.0123\end{array}$ & $\begin{array}{l}1.79 \times 10^{-2} \\
0.346 \\
9.7 \times 10^{-3} \\
8.4 \times 10^{6} \\
65200 \\
8.59 \\
0.114 \\
0.0172\end{array}$ & $\begin{array}{l}2.54 \times 10^{-2} \\
0.560 \\
0.051 \\
8.3 \times 10^{6} \\
54000 \\
9.67 \\
0.285 \\
0.0167\end{array}$ \\
\hline
\end{tabular}

plot is the envelope of the maximum heat transfer rate recorded at each circumferential location during the period of useful test flow.

\section{COMPARISON WITH PREDICTED HEAT TRANSFER RATES}

The measured heat transfer rates may be reduced to Stanton number form and compared with the established theoretical prediction of stagnation point heat flux due to Fay and Riddell. ${ }^{10}$ Only a summary treatment is possible here. The reader is referred to Sanderson ${ }^{11}$ and forthcoming publications by the authors for additional detail. When the stagnation point boundary layer is in chemical equilibrium, computations of the heat flux are correlated by an expression of the form ${ }^{10}$

$$
\begin{aligned}
\dot{q}= & \zeta\left(\rho_{w} \mu_{w}\right)^{0.1}\left(\rho_{e} \mu_{e}\right)^{0.4} \sqrt{\left.\frac{d u_{e}}{d x}\right|_{0}}\left(h_{e}-h_{w}\right) \\
& \times\left[1+\left(\mathrm{Le}^{0.52}-1\right) \frac{h_{d}}{h_{e}}\right] .
\end{aligned}
$$

Here $\dot{q}$ is the heat transfer per unit area and time, $\rho$ is the gas density, $\mu$ is the viscosity, $d u /\left.d x\right|_{0}$ is the transverse velocity gradient at the outer edge of the boundary layer, and Le is the Lewis number. The subscripts, $w, e$, and $\infty$ denote conditions at the wall, at the outer edge of the boundary layer, and in the free stream, respectively. Variations in enthalpy $h$ are de- scribed with the same subscripts and $h_{d}$ is the chemical component of enthalpy for the dissociated gas at the edge of the boundary layer. The constant of proportionality is $\zeta=0.94$ for axisymmetric bodies. For planar bodies the value $\zeta$ $=0.70$ is obtained by extrapolation of results for nonreacting flow. ${ }^{12}$ Expressing the heat flux as a Stanton number we have,

$$
\mathrm{St}=\frac{\dot{q}}{\rho_{\infty} u_{\infty} h_{0 \infty}} .
$$

The small wall enthalpy has been neglected in the definition of the Stanton number for hypervelocity flow. Invoking the Newtonian approximation for thin shock layers, Lighthill's model $^{13}$ for the caloric and thermal equations of state of an ideal dissociating gas, $\mathrm{Le}=1.4$, a power law temperature dependence of viscosity, $\mu \sim T^{0.7}, T_{w} \ll T_{e}$, and assuming recombination at the wall we obtain

$$
\begin{aligned}
\mathrm{St}= & 2^{3 / 4} \zeta \operatorname{Re}_{\infty}^{-1 / 2}\left(\frac{1+\alpha_{\infty}}{P_{\infty}}\right)^{0.38} \\
& \times\left(1+\alpha_{e}\right)^{-0.28} \hat{T}_{w}^{-0.03} \hat{\rho}_{e}^{-0.13}\left[1+0.2 \frac{\alpha_{e}}{H_{0_{\infty}}}\right] .
\end{aligned}
$$

Here $\alpha$ denotes the dissociated mass fraction with subscripts as before. The Reynolds number is defined with respect to the body diameter $D$ and the free-stream conditions $\operatorname{Re}_{\infty}$ $=\rho_{\infty} u_{\infty} D / \mu_{\infty}$. The dimensionless stagnation enthalpy $H_{0_{\infty}}$ is 

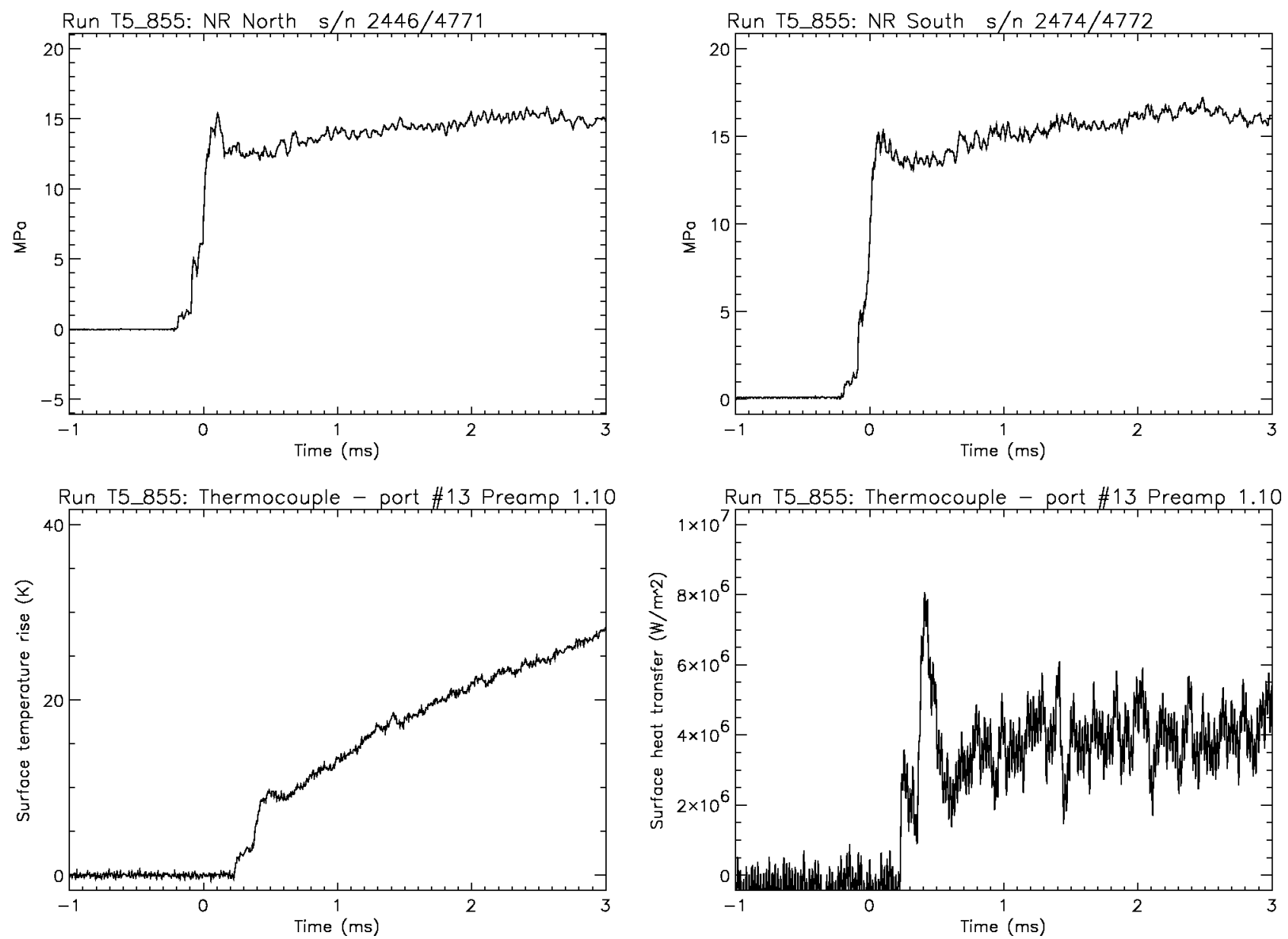

FIG. 5. Temporal characteristics of heat transfer measurement in a hypervelocity shock tunnel. Upper plots show the nozzle reservoir pressure. Lower plots show the stagnation point surface temperature and the heat flux inferred from it.

normalized with respect to the activation energy for the dissociation reaction $H_{0_{\infty}}=2 m h_{0_{\infty}} / k \theta_{d}$, where $k$ is Boltzmann's constant, $m$ is the mass of one atom of the gas, and $\theta_{d}$ is a temperature characterizing the dissociation energy. Generally the notation $\hat{\phi}=\phi / \phi_{\infty}$ is used to describe a quantity normalized with respect to its value in the free stream. The dimensionless parameter $P_{\infty}=p_{\infty} / \rho_{\infty} u_{\infty}^{2}$ arises and it behaves as $P_{\infty} \sim 1 / M_{\infty}^{2}$ for a perfect gas. The remaining variables $\alpha_{e}$ and $\hat{\rho}_{e}$ are constrained by the standard conservation equations solution for the normal shock wave ahead of the blunt body. In the current notation this reduces to

$\left(H_{0_{\infty}}-\alpha_{e}\right) \hat{\rho}_{e}^{2}-2 K_{\infty}\left(1+P_{\infty}\right) \frac{4+\alpha_{e}}{1+\alpha_{e}} \hat{\rho}_{e}+K_{\infty} \frac{1+\alpha_{e}}{1+\alpha_{e}}=0$.

The solution is closed by invoking the law of mass action for equilibrium of the dissociated gas

$$
\frac{\alpha_{e}^{2}}{1-\alpha_{e}}=\frac{\hat{\rho}_{d}}{\hat{\rho}_{e}} \exp \frac{\left(1+\alpha_{e}\right) \rho_{e}^{2}}{2 K_{\infty}\left(1-\hat{\rho}_{e}\left(1+P_{\infty}\right)\right)} .
$$

An additional dimensionless equilibrium constant is introduced, $\hat{\rho}_{d}=\rho_{d} / \rho_{\infty}$, and

$$
K_{\infty}=\frac{m u_{\infty}^{2}}{k \theta_{d}}=\frac{H_{0_{\infty}}-\alpha_{\infty}}{1+2 P_{\infty} \frac{4+\alpha_{\infty}}{1+\alpha_{\infty}}} .
$$

Variation of the flow variables as the subsonic flow decelerates from postshock conditions to the stagnation point is neglected since the postshock Mach number is low. Simultaneous solution of Eqs. (16), (17), and (18) results in the predicted stagnation point Stanton number $\operatorname{St}\left(P_{\infty}, H_{0_{\infty}}, \alpha_{\infty}, \hat{\rho}_{d}, \operatorname{Re}_{\infty}, \hat{T}_{w}\right)$ for hypervelocity flow of a dissociating gas about a blunt body.

The experimental data are compared with the predicted heat fluxes in Fig. 6 by normalizing the data with respect to the Stanton number predicted by Eq. (16). Numerical values for the predicted Stanton number at each test condition are given in Table I.

\section{ERROR SOURCES}

The performance demonstrated above was obtained repeatably and without difficulty after initial development of the gauge. Noise levels in the measured surface temperature were acceptably low. Noise levels in the deconvolved heat transfer rate were determined by the characteristics of the least squares optimal deconvolution approach that balances the suppression of signal noise with sensible time dependent information extraction (Fig. 5). The transducer set was used 

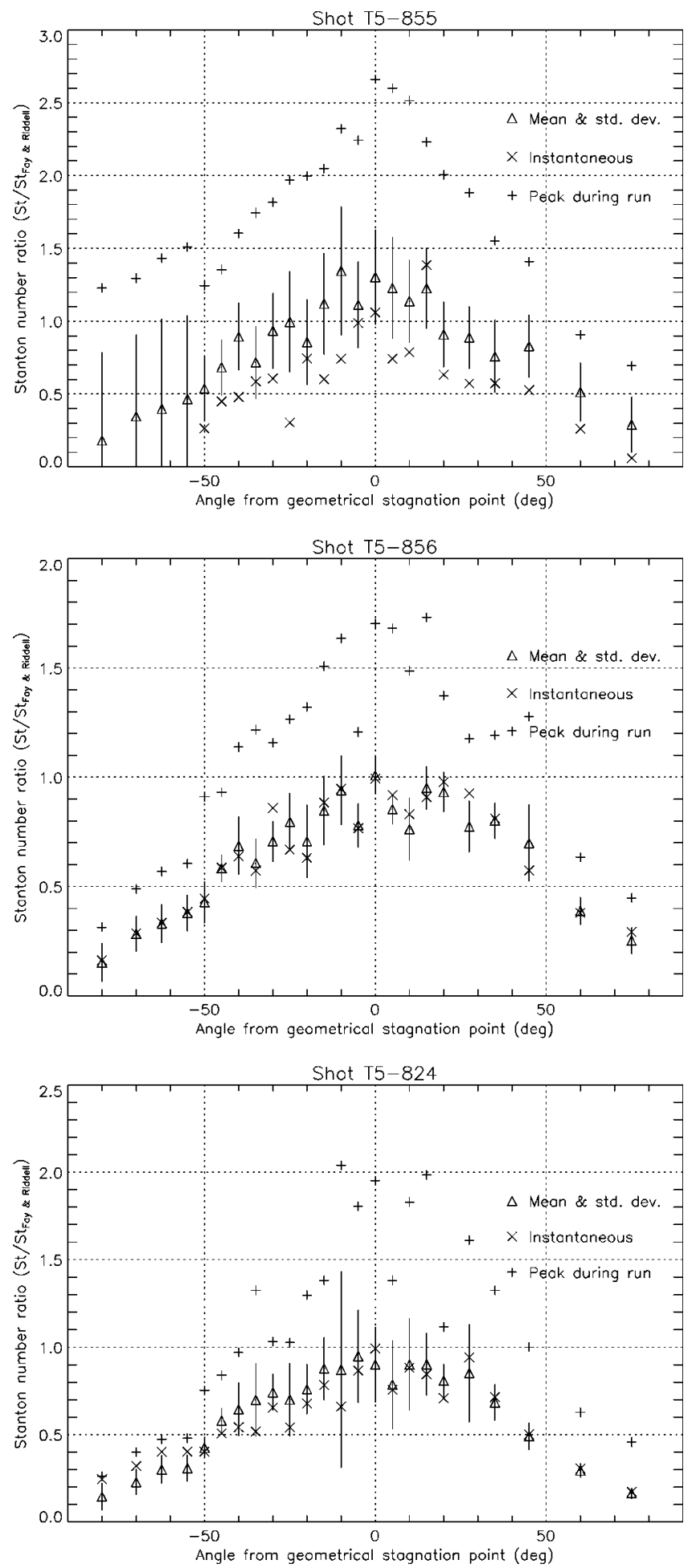

FIG. 6. Distribution of Stanton number about the forebody of a cylinder in hypervelocity flow. (Top-bottom) test conditions A, B, and C as shown in Table I. Data are normalized with respect to the stagnation point heat flux predicted by Fay and Riddell (Ref. 10).

for approximately 40 shots of the T5 hypervelocity shock tunnel at GALCIT. At the end of the test sequence, no transducer failures or performance degradation had been observed.
Note that the thermocouples are symmetrically located at several locations $\left( \pm 5^{\circ}, \pm 10^{\circ}, \pm 15^{\circ}, \pm 20^{\circ}, \pm 35^{\circ}\right.$, and $\pm 45^{\circ}$; see Fig. 6). Formal one-way analysis of variance on this replicated data set for test condition A yields a residual standard deviation of $8.9 \%$ with respect to the unit heat transfer rate predicted by Fay and Riddell, ${ }^{10}$ and a $99.6 \%$ confidence level that variations in heat flux about the model forebody can be resolved. Note that the error sources pooled in this analysis include gauge fabrication, material property variations, signal path variability, and shock tunnel flow asymmetry. We assert that this residual variance, along with variance in the predicted test conditions (Table I), reconciles any discrepancies observed in the heat flux data (Fig. 6) relative to the established predictions of Fay and Riddell. ${ }^{10}$

Further errors are associated with the uncertainty in the physical properties of the thermocouple elements, the difference in $\alpha$ and $k$ for the two dissimilar metal conductors, and the reduced heat conduction away from the surface due to tapering of the center conductor may be eliminated by calibration. The tapering of the Constantan pin effectively reduces its thermal diffusivity and this error tends to negate the unavoidably lower thermal diffusivity of Chromel. The remaining systematic error due to the finite thickness of the surface junction may be estimated from the preceding analysis. Calibration allows reduction of these error sources to any desired level of accuracy (e.g., Lyons and $\mathrm{Gai}^{2}$ ).

Under test conditions more extreme than those reported here, the physical properties of the thermocouple materials change significantly with temperature. Particularly strong variations of the specific heat are possible in the vicinity of solid state phase transitions. Metastable phases that may arise under conditions of rapid heating and cooling pose further difficulties. Existing analyses for deducing the model heat transfer rate from the surface temperature time history are based on linear hypotheses. Some literature exists for estimating the errors associated with the quasilinear temperature dependence of the material properties (e.g., Hartunian and Varwig ${ }^{14}$ ). The issue of strong nonlinearity associated with phase transitions remains open.

${ }^{1}$ D. L. Schultz and T. V. Jones, AGARDograph No. 165 (1973).

${ }^{2}$ P. R. A. Lyons and S. L. Gai, J. Phys. E 21, 445 (1988).

${ }^{3}$ A. Papoulis, Probability, Random Variables and Stochastic Processes (McGraw-Hill, New York, 1984).

${ }^{4}$ W. H. Press, B. P. Flannery, S. A. Teukolsky, and W. T. Vetterling, Numerical Recipes in C (Cambridge University Press, Cambridge, 1988).

${ }^{5}$ S. R. Sanderson and J. M. Simmons, AIAA J. 29, 2185 (1991).

${ }^{6}$ R. J. Stalker, AIAA J. 5, 2160 (1967).

${ }^{7}$ H. G. Hornung, B. Sturtevant, J. Belanger, S. R. Sanderson, M. Brouillette, and M. Jenkins, Proceedings of the 18th International Symposium on Shock Waves, 21-26 July, Sendai, Japan, Vol. 1, pp. 603-610.

${ }^{8}$ J. A. Lordi, R.E. Mates, and J. R. Moselle, NASA Report CR-472, 1966.

${ }^{9}$ M. K. McIntosh, Department of Physics Internal Report, School of General Studies, Australian National University, Canberra, 1968.

${ }^{10}$ J. A. Fay and F. R. Riddell, J. Aeronaut. Sci. 25, 73 (1958).

${ }^{11}$ S. R. Sanderson, Ph.D. thesis, California Institute of Technology, 1995.

${ }^{12}$ F. M. White, Viscous Fluid Flow (McGraw-Hill, New York, 1974).

${ }^{13}$ M. J. Lighthill, J. Fluid Mech. 2, 1 (1957).

${ }^{14}$ R. A. Hartunian and R. L. Varwig, Phys. Fluids 5, 169 (1962). 